\title{
Adaptive Five-State based Sensor Scheduling for Energy Conservation in Wireless Sensor Networks
}

\author{
P. Leela Rani \\ Assistant Professor, Department of Information \\ Technology, \\ Sri Venkateswara College of Engineering, Pennalur \\ Village, Chennai-Bengaluru High Road, \\ Sriperumbudur Tk., Kancheepuram Dist.,Chennai \\ 602117, India
}

\author{
G.A. Sathish Kumar, $\mathrm{PhD}$ \\ Professor, Department of Electronics and \\ Communication Engineering, \\ Sri Venkateswara College of Engineering, Pennalur \\ Village, Chennai-Bengaluru High Road, \\ Sriperumbudur Tk., Kancheepuram Dist.,Chennai \\ 602117, India
}

\begin{abstract}
Target tracking is the trending topics of research in Wireless Sensor Networks. It deals with detecting and estimating the consecutive positions of single or multiple targets during their course of movement in the observed area. Reducing the expenditure of energy, while offering high precision in tracking, is a tricky problem, as sensor nodes are restricted in terms of energy. The sensor nodes may be made to sleep to conserve energy. Nevertheless, sleep scheduling amplifies the likelihood of target loss while tracking, when the sensor nodes that are supposed to be active, are asleep. Consequently, there is a tradeoff between target coverage and efficiency in terms of energy. In this paper, we propose an adaptive five-state based sensor scheduling that balances this tradeoff. Simulation results illustrate that this scheme leverages between energy conservation and coverage efficiently.
\end{abstract}

\section{General Terms}

Wireless Sensor Networks, target tracking.

\section{Keywords}

Target tracking, wireless sensor networks, sleep scheduling

\section{INTRODUCTION}

Wireless Sensor Networks (WSNs) contain a great number of sensor nodes that position themselves into multi-hop radio networks. The nodes are usually operated by power constrained batteries. These batteries are overpriced and difficult to be changed, after deployment of the network. Hence, this factor is a critical concern to reduce energy consumption in the design of network. Previous work [1], [2] has revealed that the majority of energy is expended in listening state. As a matter of fact, it expends approximately the same level of energy, needed for receiving. Hence, nodes are normally planned to be put to sleep when no other activity is progressing [3]. Nodes could be made to function in a low duty cycle mode, after sleep scheduling, which can be initiated once in a while to confirm the occurrence of any activity in the channel. Keshavarzian et al. [4] examined diverse schemes for sleep scheduling and proposed a scheduling technique to reduce the delay. However, this technique is not interference-free. Chao Gui and Prasant Mohapatra [5] proposed a wake up scheme for tracking. In this paper, an adaptive five-state based sensor scheduling approach is proposed that performs pre scheduling of sleeping and hence avoids interference.

The main contributions of this paper are summarized as follows:

(1) Pre scheduling based sleeping mechanism
(2) Adaptive five-state based sensor scheduling approach

(3) Results of performance evaluation with respect to energy conservation and coverage of target.

The remainder of this paper is structured as follows. Related work is described in Section 2. Section 3 describes the system model. Section 3 analyzes the results for the proposed model. Section 4 presents the conclusion and future work.

\section{RELATED WORK}

A given area that should be monitored would function entirely with sensor nodes that cover the area with surplus power. However, the sensor nodes have restricted energy; the accuracy of tracking is considered as inversely proportional to the network life time. There is always a trade-off between energy conservation and accuracy of target tracking in WSNs. A sleep plan must be made for each sensor node as a chart for deciding when to turn the sensor nodes into the sleep mode. The more each sensor node is in the sleep mode, the energy expended per unit time is less for the network. Hence, it is mandatory that the distribution and the number of the sensor nodes that are active in the sensing field at any given time are scheduled. However, if less number of sensors alone is awakened to track the target, it might affect the tracking accuracy. A compromise must be reached regarding the number of sensors to be used for tracking and the energy expended during tracking. Many research works on coverage [5] stressed on full coverage in the field of sensing. However, the cost of deployment, the physical restrictions, and the efficiency of functioning recommend using a limited coverage of the sensing field. The trade-offs between energy saving and the accuracy of tracking are extensively researched. Various works confirm that major energy saving can be achieved while reducing the error of tracking within tolerable limits. The coverage of WSN influences the accuracy of tracking in the sensing field. Cardei and $\mathrm{Wu}[6]$ have surveyed several coverage models and solutions. The approximation based centralized technique finds the minimum sensor nodes to cover each and every position in the sensing field. The integer programming based centralized techniques also finds the minimal set of sensor nodes that can cover maximum locations in the sensing field. Huang and Tseng [7] offer a solution to check the network coverage. The condition for network coverage is that every position of monitoring field should be enclosed with in at least k sensors. Wang et al. [8] considered the relationship between coverage and connectivity. They assumed different associations between the ranges of sensing and communication for every node. It is obvious that a moderately covered sensing region will perform in a better way in detecting the incidence of a moving 
target, while achieving considerably superior energy savings compared to a completely covered sensing field. Applications for tracking targets are an instance to rationalize the requirement for exploring moderately covered sensing field. Given a WSN composed of sensors, accuracy of tracking is determined by the least and greatest coverage path within the sensing field. The least coverage path is used to discover the least monitored region of the sensing field. These are first recognized as a metric for assessing quality for tracking targets in sensor networks [9]. Maximum breaches expose the weakest observed region of the sensing field and hence offers most favorable navigation for the target. But it does not throw light on the concentration and spreading of sensor nodes for target detection. In applications related to tracking targets, the targets that are being monitored and sensed are mobile within the sensing field. The motion of the targets suggests that every position of the sensing field need not be covered. A sensor network with small void areas can detect a target in motion almost immediately with a small lag. This supports the notion that more and more sensor nodes can be put in sleep mode. This idea offers maximum coverage and saves energy simultaneously, in comparison to having all the sensor nodes awake for full target coverage.

An adaptive sleep scheduling mechanism is proposed in this paper, taking into consideration both energy conservation and target coverage.

\section{SYSTEM MODEL}

The sensor nodes deployed in a given sensing field is represented as spatial points. The deployed sensor nodes are denoted using $\left\{\mathrm{s}_{1}, \mathrm{~s}_{2}, \cdots, \mathrm{s}_{\mathrm{k}}\right\}$ in a bounded area $\mathrm{Y} . \xi_{\mathrm{i}}$ denotes the position of $s_{i}$ in $X$. When $n$ is large and the events are spread autonomously and regularly within $\mathrm{X}$, then the process is considered as homogeneous Poisson. At any instant of time, sensor node $\mathrm{N}_{\mathrm{i}}$ situated at $\xi_{\mathrm{i}}$ has a sensing range of $\mathrm{r}_{\mathrm{i}}$. The sensing range is denoted by the disc as given in equation (1).

$$
\xi_{\mathrm{i}}+\mathrm{T}\left(\mathrm{r}_{\mathrm{i}}\right)
$$

Hence, the coverage of the sensing field is defined as a series of arbitrary discs, which is denoted as D as given in equation (2).

$$
\mathrm{D} \equiv\left\{\xi_{\mathrm{i}}+\mathrm{T}\left(\mathrm{r}_{\mathrm{i}}\right), 1 \leq \mathrm{i} \leq \mathrm{k}\right\} .
$$

A point $\mathrm{w}$ is considered to be covered by the coverage if it is present in at least one of the discs as shown in equation (3).

$$
\xi_{\mathrm{i}}+\mathrm{T}\left(\mathrm{r}_{\mathrm{i}}\right)
$$

The sensing ranges of the $\mathrm{k}$ nodes, $\left\{\mathrm{r}_{1}, \mathrm{r}_{2}, \cdots, \mathrm{r}_{\mathrm{k}}\right\}$, are $\mathrm{n}$ autonomously and identically spread random variables. The values of the random variables are in the range $\left[r_{\min }, r_{\max }\right]$ with mean $\overline{\mathrm{r}}$.

\subsection{Target tracking}

The formal definition for the problem of target tracking assumes that a target is deployed at a random time at an arbitrary position within the sensing field, and it disappears after travelling for some duration. In equation (4), $\mathrm{E}^{*}$ is the expected duration of travel by the target before it is detected.

$$
\mathrm{m}=\frac{1}{\mathrm{E}^{*}}
$$

The amount of time spent before the target is tracked is influenced by $\mathrm{m}$ and the target's velocity. Sensor nodes distribution pattern are of two groups: the homogeneous distribution and the planned distribution. The deployment of sensor nodes may not be homogeneous due to various limitations in physical world. This non homogeneous distribution can be adjusted by sleep planning. This helps to attain a homogeneous distribution of active sensor nodes. In sleeps scheduling, only the sensor nodes at intended positions are active. Planned coverage guarantees more accurate tracking.

\subsection{Pre-scheduled sleeping mechanism of deployed sensors}

Let us assume a sensor network which is distributed and is used for monitoring an operational area. The functioning of the sensor network has two modes, namely the observation mode during the absence of any interesting event, and the tracking mode in which the response to any target in motion is provided. The sensor node should, at the outset, operate in the low power mode when the sensor network is devoid of any target. The sensor node should quit the low power mode and switch to being active seamlessly for certain duration during which a target come into its sensing range, or when a target is about to enter. Lastly, when the target travels farther away from the sensor node, the node should make a decision to toggle back to the low power state. This allows each deployed sensor node, during network initialization, to formulate its own sleep schedule. During the functioning, each sensor node separately follows its own sleep plan, without cooperating with each other. The basic pre-scheduled sleeping is executed by a random scheme. Each sensor node divides the time into equal length slots; $\mathrm{T}_{\mathrm{s}}$. The period of each $\mathrm{T}_{\mathrm{s}}$ is separated into two components namely, the active and the sleeping period. The period of active duration is $\mathrm{k} * \mathrm{~T}_{\mathrm{s}}$. The sleeping period occupies the rest of $T_{S}$. Arbitrariness is included to the margins of $T_{S}$ of all the nodes. The starting point of the $T_{s}$ of the entire sensor nodes should be arbitrarily and separately distributed. The asynchronization of clocks present at the sensor nodes adds this randomness. In random independent sleeping, each sensor node is active with a probability of $\mathrm{p}$ at any instant. The anticipated number of active sensor nodes within the area $\mathrm{X}$ at any instant is $\mathrm{p} * \mathrm{~N}$, and the small section of active nodes are spread regularly within $\mathrm{X}$. The probability of each node being active, $p$, is given by equation (5).

$$
\mathrm{p}=\frac{\mathrm{n}^{*}}{\mathrm{~N}}
$$

where $\mathrm{n}^{*}$ is the decided active node count.

In order to decide the subset of sensors that should be awakened and made working, cooperation among neighboring nodes is needed to draft the sleeping plan. In this approach, at the outset each node is made to sleep for a different exponential duration.

The probability density function is given by equation (6).

$$
\mathrm{f}\left(\mathrm{t}_{\mathrm{s}}\right)=\lambda \mathrm{e}^{-\lambda \mathrm{t}_{\mathrm{s}}}
$$

where $\lambda$ is the rate of querying and $t_{s}$ is the duration of sleeping time. When a node awakens, it broadcasts a probe message and it starts querying the neighboring nodes in the network. The message has a controlled transmission power so that the message arrives at the preferred transmission range. Sensor nodes that are currently working send a reply message, upon receiving the probe. The sensor node that currently sent the probe message will wait for some fixed duration to gather all the reply messages from the other nodes. If there are no other awakened nodes in the network, the sensor node that sent the query message will awaken. Or else, it will return back to the sleep mode for some more duration. The duration of the next sleep is based on adaptive sleep which uses the 
information composed during the querying.

The density of awakened sensor nodes can be controlled by modifying the range of probing. One scenario here is that, an awakened node can be made to remain awake seamlessly until it turns faulty or depletes its energy. The other sensor nodes that have been sleeping would substitute any faulty sensor nodes as and when required. This is not preferable as the number of awakened nodes may get decreased as the simulation progresses. Also as a result of node failures, the network may be partitioned or the nodes may be isolated. In this scenario the energy expended should be distributed among the sensor nodes in the network. In order to share the energy consumption among sensor nodes, a node should be shifted to sleep mode after being active for some amount of time and the other neighboring nodes may be given a chance to work in the active mode. The duration of the sensor node's next sleep is set as the least value of timers received from all the other nodes. This ensures that when an active sensor node starts sleeping, other sleeping sensor nodes in the set up will wake up and start probing the environment. Multiple neighboring sensor nodes around an active node might know its next sleep duration. Hence, all these sensor nodes might wake up simultaneously and start sending the query messages. To avoid this, an arbitrary value is chosen as offset value and added to the timer of the enquiring node's wake up schedule.

\subsection{Adaptive five-state based sensor scheduling}

This section describes the sleep schedule of sensor nodes during the tracking mode. A sensor node enters the tracking mode and remains in track mode if a target is sensed during the wake up duration. A target might have crossed a sensor node during its sleep without being detected. Hence, it is essential that each sensor node must be educated regarding the movement of a target in its direction. Each sensor node can be made to operate in five functioning states namely wait, stand by, in-track, out-track and track.

The wait state is similar to the low power mode in observation mode. The sensor nodes are considered as active if they are in ready and in-track modes. Figure 1 shows the distribution of sensor nodes around the target. At any instant, any sensor node that is enclosed within the circle, that is having the present position of the target as the center and average sensing range as radius $r$, should be in track mode. It actively engages in coordinative tracking along with remaining sensor nodes in the circle. The nodes exchange track messages among the neighbors. All sensor nodes that are in active mode but lie within the range of transmission can receive and locate the packets. If any sensor node collects tracking messages but does not sense any target, it should be watchful that a target may be approaching in the near future. The sensor node may also get a choice regarding the present position and velocity of the target.

The sensor nodes, after receiving messages, move to in-track state from wait state or standby state. At the border line, an intrack sensor node can be $r+\left(\frac{R}{2}\right)$ distance farther away from the target. A sensor node also sends a ready message. Any sensor node that gets a ready message should move to standby state from wait state. A sensor node in standby state is $\mathrm{r}+2 * \mathrm{R}$ farther away from the target. A sensor node in intrack would be at $\mathrm{r}+\mathrm{R}$ distance away from the target. If a sensor node is in -track state and it no longer senses the target for a smaller duration of $\Delta \mathrm{T}$, then it moves to the track state. After $\Delta \mathrm{T}$ seconds, if it still doesn't sense the target, then it moves to out-track state. If the sensor node confirms that it is no longer getting any track messages, then it shifts to the standby mode. If the sensor node verifies that it is not receiving track or standby messages, it moves back to the wait state. Hence, a sensor node currently in track mode steadily shifts back to low power monitoring mode when the target is not in its close proximity.

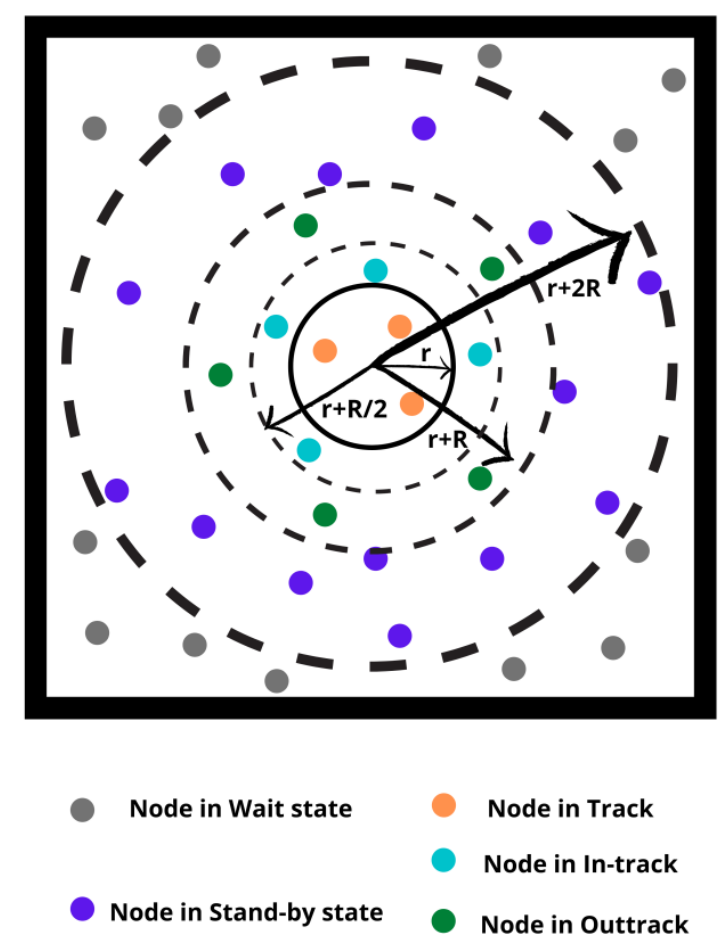

Figure 1. Five states for scheduling sensors

\subsection{Hidden markov model to model state transitions}

The transition between the states is modeled as a Hidden Markov Model (HMM) [10] as shown in figure 2. The matrix with transition probability is denoted by $\mathrm{M}(\mathrm{k})$, depending on transitions and the period of stay in each state. In HMM model, the likelihood of remaining in state $i$ for a period $t$ is given by $\mathrm{p}(\mathrm{t})=\left(\mathrm{a}_{\mathrm{ii}}\right)^{\mathrm{t}-1}\left(1-\mathrm{a}_{\mathrm{ii}}\right)$. The expected duration is $\left.E[t]=\sum_{t=1}^{\infty} t\left(a_{i i}\right)^{t-1}\left(1-a_{i i}\right)=\frac{1}{\left(1-a_{i i}\right)}\right)$.

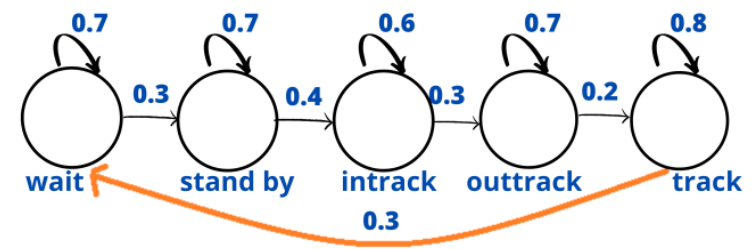

Figure 2. Five states as modeled by HMM

\section{PERFORMANCE EVALUATION}

The sensing field is a free space of size $400 \mathrm{~m} \times 400 \mathrm{~m}$. The sensor nodes are uniformly distributed and are 900 in number. The target is made to come into view in the sensing field at an arbitrary position. The target moves in random direction and follows a straight line path in that direction, with a velocity of $10 \mathrm{~m} / \mathrm{s}$. The simulation is halted when the deployed target is first located by a sensor node. The simulation is rum many times with various arbitrary target positions and travelling directions. The value of distance traveled by the target without 
being detected by any sensor node is marked in scenario 1 . In scenario 2 , the mechanism described above regarding sleep scheduling is simulated.

The considered network is of size $400 \mathrm{~m} \times 400 \mathrm{~m}$, with 900 uniformly spread sensor nodes. A smaller sub region is simulated in the sensing field. And the 800 sensor nodes are deployed in that sub region. The target is made to enter the sensing field and moves along sub region. Active period of the nodes are set. The percentage of time, a sensor node is awake, is changed. The active nodes at any instant cover the sensing field entirely with 900 deployed sensor nodes and active period around 0.4 . The value of time slot is varied from $0.5 \mathrm{sec}$ in increments of $0.5 \mathrm{sec}$. The value of $\mathrm{m}$ is found to be high. The active duration is set from 0.01 and as it is increased, the value of $\mathrm{m}$ is observed to increase. As the number of active nodes is increased, the coverage is also extended.

\subsection{Energy conservation}

The energy consumed during sleep mode is subtracted from the energy consumed by all the sensor nodes in the sensing field during active mode. This provides the amount of energy conserved. The percentage of energy conserved by applying sleep planning is observed. The number of average working sensors is varied by changing the sensor node active period. The results show that the energy conservation is linearly related to active period within a slot. The energy consumption is the sum of energy expended for sensing, listening and used for exchanging various messages. Modifying the time slot did not contribute to saving energy. If the value of watchfulness is less, then it leads to sleeping for a longer duration. A lengthy time slot offers great amount of energy conservation. The number of active nodes is restricted by the range of probing. The energy consumption is less for longer periods of active mode. This is possible because of coordination between the neighboring nodes, which serves to increase the active period as well as the sleep duration. When a sensor node sleeps for considerable amount of time, it results in low energy consumption due to the fact that only less number of control messages will be generated. However, the energy conserved is not much significant, throwing light on the fact that only minor amount of energy is expended for transferring control messages when compared to the energy utilized for listening and sensing. This five state approach is compared with the approach proposed by N. Essaddi, M. Hamdi and N. Boudriga [11]. The comparison in terms of energy conservation for durations of 1 second by both the approaches is shown in figure 3 .

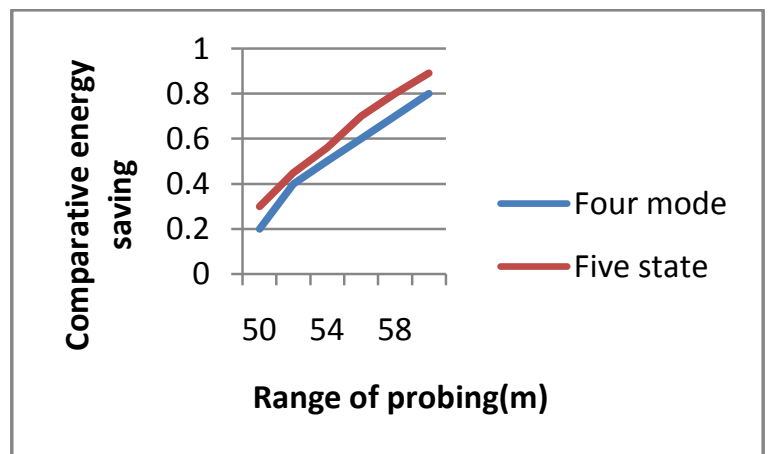

Figure 3. Energy conserved in Four mode and Five state sleep scheduling

Energy conserved for 10 seconds duration in both four mode and five state approach is displayed in figure 4.

\subsection{Target coverage}

Target coverage is the attention the target cumulatively gets when it is traveling the trajectory in the sensor field. Higher value of target coverage denotes better accuracy of tracking the target in the sensing field along the trajectory. Sleeping sensor nodes during the trajectory of a target do not add to the value of target coverage. When the value of watchfulness equals 1 , the target coverage is at its highest and the target is provided more attention along the path.

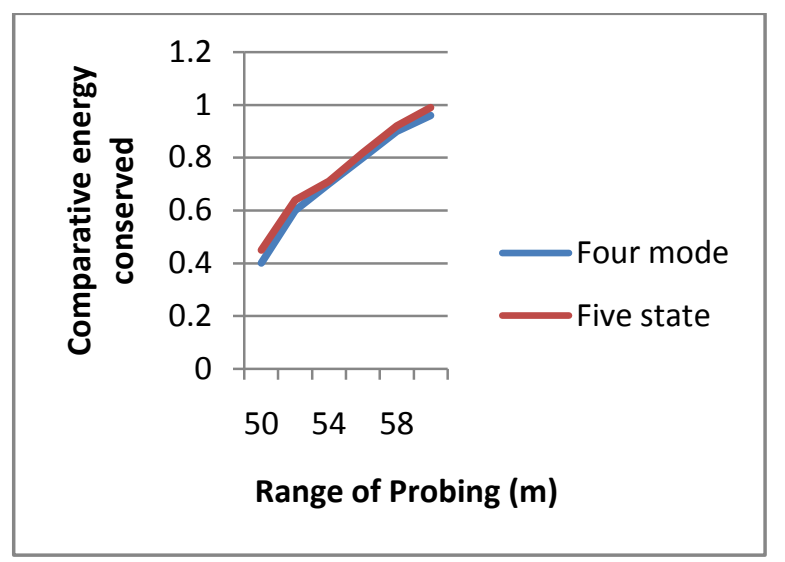

Figure 4. Energy conserved in Four mode and Five state sleep scheduling for $10 \mathrm{~s}$

As the value of watchfulness is reduced to 0.1 , the value of target coverage is decreased as it would be difficult to awaken all the sensor nodes when each sensor node is in sleeping mode most of the time. It is also observed that short time slots will improve the situation. Each sensor node will be waking up more often in short time slot; there is more possibility that a sensor node will be receiving alerts. Figure 5 displays this scenario.

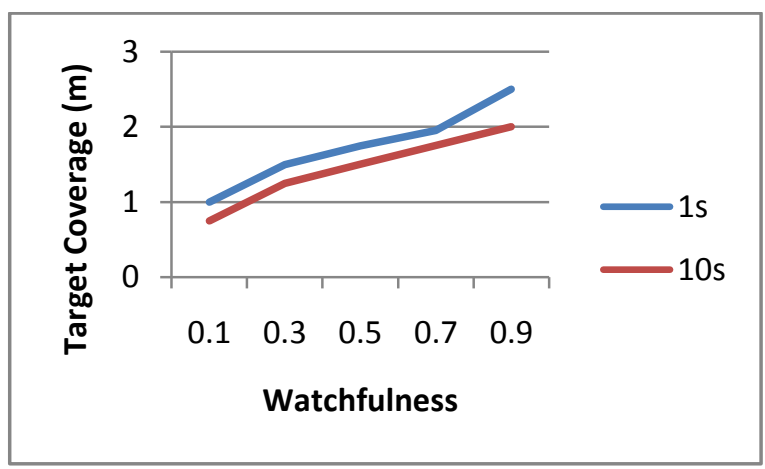

Figure 5. Target Coverage with respect to watchfulness of sensor nodes

\section{CONCLUSION}

Recent innovations in ever- evolving applications necessitate reducing the energy expenditure in WSN. To improve network lifetime and to offer target coverage simultaneously, a sleep scheduling scheme named as the adaptive five-state based sensor scheduling is proposed. This method comprises of two major phases, viz., pre-scheduling and scheduling during tracking mode. HMM is used to model the transitions between the states. From the simulation results, it is evident that the proposed approach efficiently reduces energy consumption and also offers better target coverage. In the future, sleep scheduling for heterogeneous sensor networks will be concentrated upon, with respect to application scenarios that prefer stringent coverage requirements. 


\section{REFERENCES}

[1] Mohamed Elshrkawey, Samiha M. Elsherif, M. Elsayed Wahed, An Enhancement Approach for Reducing the Energy Consumption in Wireless Sensor Networks, Journal of King Saud University - Computer and Information Sciences, Volume 30, Issue 2, 2018, Pages 259-267, ISSN 1319-1578, https://doi.org/10.1016/j.jksuci.2017.04.002.

[2] Kozłowski, A., Sosnowski, J. Energy Efficiency TradeOff Between Duty-Cycling and Wake-Up Radio Techniques in IoT Networks. WirelessPers Commun 107, 1951-1971(2019). https://doi.org/10.1007/s11277-019-06368-0.

[3] Wan, R., Xiong, N. \& Loc, N.T. An energy-efficient sleep scheduling mechanism with similarity measure for wireless sensor networks. Hum. Cent. Comput. Inf. Sci. 8, 18 (2018). https://doi.org/10.1186/s13673-0180141-x.

[4] Abtin Keshavarzian, Huang Lee, and Lakshmi Venkatraman. 2006. Wakeup scheduling in wireless sensor networks. In Proceedings of the 7th ACM international symposium on Mobile ad hoc networking and computing (MobiHoc '06). Association for Computing Machinery, New York, NY, USA,322-333. https://doi.org/10.1145/1132905.1132941

[5] Chao Gui and Prasant Mohapatra. 2004. Power conservation and quality of surveillance in target tracking sensor networks. In Proceedings of the 10th annual international conference on Mobile computing and networking (MobiCom '04). Association for Computing Machinery, New York, NY, USA, 129-143. https://doi.org/10.1145/1023720.1023734.
[6] A. Singh and T. P. Sharma, "A survey on area coverage in wireless sensor networks," 2014 International Conference on Control, Instrumentation, Communication and Computational Technologies (ICCICCT), 2014, pp. 829-836, doi: 10.1109/ICCICCT.2014.6993073.

[7] Mihaela Cardei, Jie Wu, Energy-efficient coverage problems in wireless ad-hoc sensor networks, Computer Communications, Volume 29, Issue 4, 2006, Pages 413420, ISSN 0140-3664, https://doi.org/10.1016/j.comcom.2004.12.025.

[8] Huang, CF., Tseng, YC. The Coverage Problem in a Wireless Sensor Network. Mobile Netw Appl 10,519528 (2005). https://doi.org/10.1007/s11036-005-1564-y.

[9] Wang, Xiaorui \& Xing, Guoliang \& Zhang, Yuanfang \& Lu, Chenyang \& Pless, Robert \& Gill, Christopher. (2003). Integrated Coverage and Connectivity Configuration in Wireless Sensor Networks. SenSys'03: Proceedings of the First International Conference on Embedded Networked Sensor Systems. 1. 28-39. $10.1145 / 958491.958496$.

[10] Woosun An, Satnam Singh, Krishna R. Pattipati, David L. Kleinman, Swapna S. Gokhale: Dynamic Scheduling of Multiple Hidden Markov Model-Based Sensors. J. Adv. Inf. Fusion 3(1): 33-49 (2008).

[11] N. Essaddi, M. Hamdi and N. Boudriga, "Efficient coverage criterion for accurate target tracking using cooperative wireless sensor networks," 2009 International Conference on Ultra Modern Telecommunications \& Workshops, 2009, pp. 1-8, doi: 10.1109/ICUMT.2009.5345383. 